\title{
CLASSIFICAÇÃO DE BIODIESEL NA REGIÃO DO VISÍVEL
}

\section{Germano Véras*, Anna Luiza Bizerra de Brito, Adenilton Camilo da Silva, Priscila da Silva, Gean Bezerra da Costa e Lorena Cristina Nóbrega Félix}

Departamento de Química, Centro de Ciências e Tecnologia, Universidade Estadual da Paraíba, 58429-500 Campina Grande PB, Brasil

David Douglas de Sousa Fernandes e Marcelo Marques de Fontes

Departamento de Ciências Agrárias, Universidade Estadual da Paraíba, 58.429-500 Campina Grande - PB, Brasil

Recebido em 17/3/11; aceito em 15/8/11; publicado na web em 26/9/11

\begin{abstract}
BIODIESEL CLASSIFICATION IN THE VISIBLE REGION. Classification of biodiesel by oilseed type using pattern recognition techniques is described. The spectra of the samples were performed in the Visible region, requiring noise removal by use of a first derivative by the Savitzky-Golay method, employing a second-order polynomial and a window of 21 points. The characterization of biodiesel was performed using HCA, PCA and SIMCA. For HCA and PCA methods, one can observe the separation of each group of biodiesel in a spectral region of 405-500 nm. SIMCA model was used in a test group composed of 28 spectral measurements and no errors are obtained.
\end{abstract}

Keywords: biodiesel; classification; visible region.

\section{INTRODUÇÃO}

Nas últimas décadas cresceu o interesse por combustíveis renováveis, em contraposição aos derivados do petróleo, ${ }^{1}$ de custo cada vez mais elevado por serem prospectados em áreas com acesso cada vez mais difícil e necessitando de maiores esforços tecnológicos. No Brasil, a produção de biocombustíveis surgiu como uma alternativa para diversificação da matriz energética, contribuindo para a preservação do meio ambiente, ${ }^{1,2}$ e para diminuir a dependência de uma fonte energética esgotável, o petróleo, apesar dos esforços em pesquisas de novos campos petrolíferos, como a descoberta de grandes reservas na camada do pré-sal.

No Brasil dois biocombustíveis se destacam: o bioálcool, produzido a partir da cana-de-açúcar, com o qual o país detém tecnologias avançadas em toda a cadeia produtiva; e o biodiesel, introduzido na matriz energética brasileira pela Medida Provisória $n^{\circ} .214$, de 13/9/2004. ${ }^{2}$ Nesta norma, o biodiesel, combustível composto de monoalquilésteres de ácidos graxos de cadeia longa, é conceituado como "um combustível para motores a combustão interna com ignição por compressão, renovável e biodegradável, derivado de óleos vegetais ou de gorduras animais, que possa substituir parcial ou totalmente o óleo diesel de origem fóssil". ${ }^{2}$

O biodiesel pode ser obtido por diferentes processos, dentre os quais se destaca a transesterificação de óleo vegetal ou gordura com um álcool de baixo peso molecular, como etanol ou metanol, através de uma reação catalisada por hidróxido de potássio ou de sódio. ${ }^{3}$

Apesar do diesel de petróleo comercializado ter $5 \%(\mathrm{v} / \mathrm{v})$ de biodiesel, ${ }^{2}$ este combustível carece de métodos de classificação de sua fonte de origem, que possa permitir um acompanhamento mais rigoroso dos padrões de qualidade de produção. Como os óleos são definidos pelos triacilgliceróis presentes e suas proporções e o método padrão de identificação e quantificação é a cromatografia gasosa, que é caro e demorado, uma alternativa viável seria desenvolver um método espectroscópico que possa ser utilizado para associar o espectro com as quantidades dos triacilgliceróis e suas proporções no óleo. Na esteira deste raciocínio, alguns trabalhos são encontrados

*e-mail: germano.veras@pq.cnpq.br para reconhecimento de padrões de biodiesel por seu óleo de partida, sendo que os mesmos utilizam a região do infravermelho próximo. ${ }^{1,4}$ Entretanto, medidas espectroscópicas na região do NIR oferecem alguns inconvenientes, tais como, necessidade de correção de linha de base, custo relativamente elevado e dificuldade de aquisição de diodos emissores de luz e dos fototransistores como fotransdutores em toda a região do NIR, dentre outros.

Assim, a espectroscopia de absorção molecular na região UV-Vis, método baseado na interação da radiação eletromagnética na faixa espectral de 200 a $800 \mathrm{~nm}$ com a matéria, apresenta-se como uma alternativa viável para reconhecimento em padrões de biodiesel por permitir construção de instrumentos mais baratos e com componentes de fácil aquisição em lojas de eletrônicos mesmo em cidades de porte médio. ${ }^{5}$

Por outro lado, a complexidade de interpretação qualitativa dos espectros na região UV-Vis conduz à necessidade de utilização de ferramentas quimiométricas para reconhecimento de padrões. Diversos trabalhos foram publicados utilizando técnicas de reconhecimento de padrões, dentre os quais para amostras de vinhos, ${ }^{6,7}$ carne de porco ${ }^{8} \mathrm{e}$ óleo de oliva, ${ }^{9}$ apesar da perspectiva conceitual de que os espectros na região do visível serem pouco discriminativos. ${ }^{10}$

As técnicas de reconhecimento de padrões podem ser divididas em duas classes: não supervisionadas e supervisionadas. ${ }^{11}$ Das técnicas não supervisionadas, que têm a finalidade de verificar a formação natural de conjunto de amostras similares, ou agrupamentos, as de maior destaque são análise por componentes principais (PCA - Principal Components Analysis) e análise hierárquica de agrupamentos (HCA - Hierarchical Clusters Analysis). A técnica HCA baseia-se na similaridade e dissimilaridade entre amostras por meio do cálculo de distância entre elas. ${ }^{12}$ A técnica PCA baseia-se na manipulação matemática dos dados originais para se obter novas variáveis, que são combinações lineares das variáveis originais e que apresentam ortogonalidade entre si, denominadas de componentes principais (PCs). ${ }^{12}$ Em relação aos métodos supervisionados, que utilizam informação prévia sobre um conjunto de amostras conhecidas, a técnica mais utilizada é a modelagem suave independente de analogias entre classes (SIMCA - Soft Independent Modeling of Class Analogies), que usa PCA para cada classe definida e testa a distância das amostras 
em relação a cada uma das classes, para determinar se se situa em alguma classe e em qual ou quais delas. ${ }^{9}$

Assim, neste trabalho buscou-se classificar amostras de biodiesel em termos do tipo de óleo utilizado na produção de biodiesel (soja, algodão e girassol), utilizando espectrometria na região do visível e aplicando técnicas de reconhecimento de padrões não supervisionado e supervisionado. As técnicas não supervisionadas utilizadas foram PCA e HCA e o método supervisionado foi o SIMCA, para fazer a classificação.

\section{PARTE EXPERIMENTAL}

\section{Reagentes e amostras}

Para obtenção das amostras de biodiesel foram utilizadas oito amostras, de marcas e lotes distintos, de óleos refinados de algodão, soja, girassol, perfazendo 72 amostras. Etanol absoluto foi utilizado para promover a reação de transesterificação e como catalisador para reação foi utilizado hidróxido de potássio PA. Após a reação de transesterificação, as amostras de biodiesel foram lavadas com água destilada e solução de ácido clorídrico $0,1 \mathrm{~mol} / \mathrm{L}$, até alcançar $\mathrm{pH}$ próximo de 7,0. A lavagem do biodiesel tem a finalidade de retirar impurezas, tais como, o excesso de álcool, que dificulta a separação entre as fases biodiesel/glicerina e afeta o ponto de fulgor do combustível; glicerina, que pode formar resíduos nos tanques de armazenamento e causar entupimentos em filtros e depósitos nos bicos injetores dos automóveis; sais dos ácidos graxos, que reduzem o rendimento da reação e, o excesso do catalisador hidróxido de potássio, o qual pode ocasionar a formação de sais de ácidos graxos. ${ }^{13}$

\section{Instrumentos e programas computacionais}

As medidas espectrométricas de refletância difusa foram realizadas em triplicata em um espectrômetro Vis/NIR FOSS XDS Master$\mathrm{Lab}^{\mathrm{TM}}$. Selecionou-se a faixa de 400 a $780 \mathrm{~nm}$, com uma resolução espectral de $0,5 \mathrm{~nm}$ para posterior pré-tratamento dos dados com seleção de faixa de variáveis mais significativas para classificação.

Os dados foram tratados com o programa computacional The Unscrambler 9.8, desde o pré-processamento de amostras e de variáveis como a construção dos modelos PCA e SIMCA. O programa computacional Statistica 9.0 foi utilizado para construir o modelo HCA para verificação dos agrupamentos dos tipos de biodiesel. O programa computacional Matlab 7.11.0.584 (R2010b) foi utilizado para seleção do conjunto de classificação e de teste para construção do modelo SIMCA, utilizando o algoritmo de Kennard-Stone.

\section{RESULTADOS E DISCUSSÃO}

\section{Aquisições dos dados espectrais}

Os espectros apresentados na Figura 1a são das amostras de biodiesel de algodão, girassol e soja na região de 400 a $780 \mathrm{~nm}$. Os dados foram pré-processados por primeira derivada pelo método de Savitzky-Golay, empregando um polinômio de segunda ordem e janela de 21 pontos permitindo destacar os comprimentos de onda mais discriminativos na classificação das amostras (Figura 1b). Baseada na seleção de variáveis a priori das variáveis mais discriminativas foi escolhida a região de 405 a $500 \mathrm{~nm}$ para a construção dos algoritmos de classificação.

\section{Modelo PCA}

O conjunto de treinamento para modelo PCA foi construído com
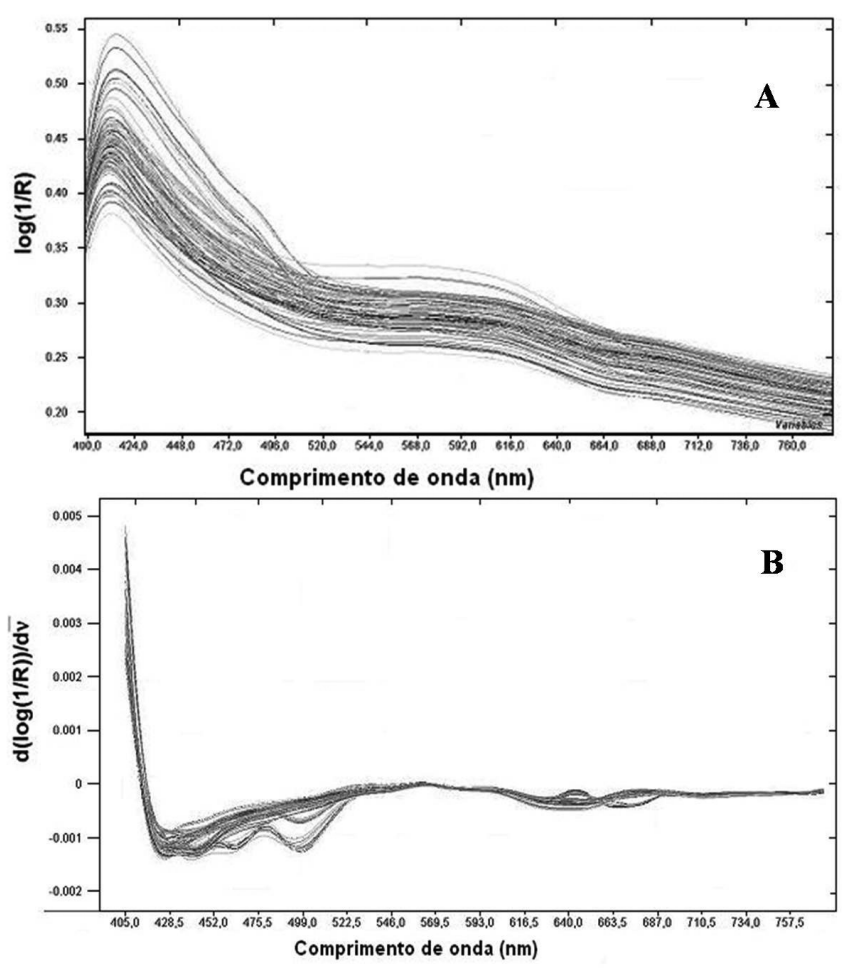

Figura 1. Espectros brutos de absorbância (A) e processados matematicamente por derivada de primeira ordem por Savitzky-Golay $(B)$ das amostras de biodiesel

as 72 amostras de biodiesel e o conjunto de validação foi baseado no procedimento de validação cruzada completa. Esta validação interna baseia-se na retirada uma amostra por vez do conjunto de treinamento e, em seguida, é testada no modelo gerado, tornando o modelo mais robusto por testar todas as amostras. A Figura 2 apresenta a distribuição de scores para as amostras de biodiesel estudadas, baseando-se em três PC, em que é possível verificar a formação de três agrupamentos, associados com o tipo de óleo utilizado na síntese das amostras de biodiesel.

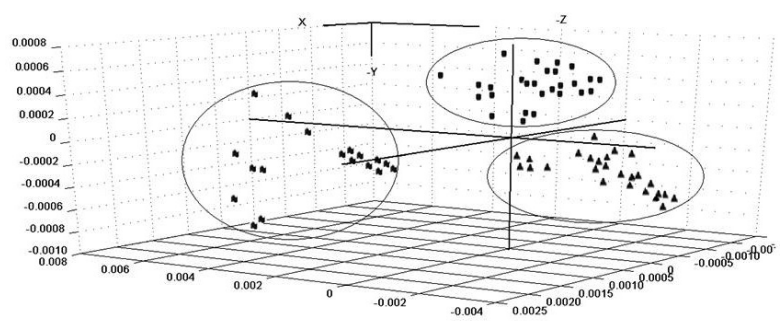

Figura 2. Distribuição de scores PC1 x PC2 x PC3 algodão, $\mathbf{\square}$ soja, A girassol)

\section{Modelo HCA}

O dendograma, apresentado na Figura 3, do modelo HCA foi construído aplicando a correlação de 1-Pearson R para todas as 72 amostras de biodiesel, que determina a extensão em que valores de duas variáveis são proporcionais entre si, para medida de dissimilaridades entre as amostras e a técnica de ligação completa, complete linkage, que indica a proximidade entre os agrupamentos das amostras por meio da regra dos vizinhos mais distantes.

Os seguintes agrupamentos HCA foram obtidos através do uso dessa técnica, baseando-se na distância de ligação máxima obtida: 


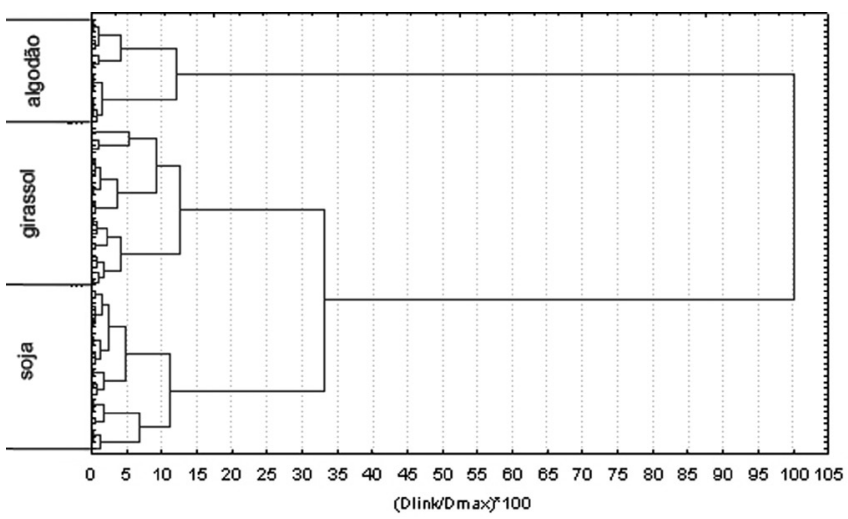

Figura 3. Dendograma obtido da análise de agrupamento hierárquico (HCA) para as amostras de biodiesel derivados de algodão, soja e girassol

agrupamento de algodão (distância de ligação de 12,0\%), agrupamento de girassol (distância de ligação de 12,5\%), agrupamento de soja (distância de ligação de 11,2\%), todos aproximadamente na mesma faixa de distância de ligação. Foi possível verificar que os agrupamentos das amostras de biodiesel se diferenciaram nas seguintes distâncias, com base na distância de ligação máxima obtida: grupamento de soja para girassol (distância de ligação de 33,2\%), grupamentos de soja e girassol para o agrupamento de algodão (distância de ligação de $100 \%$ ). Assim, o modelo HCA construído para todas as amostras de biodiesel corrobora os resultados apresentados no modelo PCA, verificando a presença de três agrupamentos também associados com o tipo de óleo utilizado na síntese das amostras de biodiesel.

\section{Modelo SIMCA}

Os conjuntos de treinamento dos modelos SIMCA para cada tipo de biodiesel (algodão, girassol e soja) foram construídos com 12 medidas espectrais do biodiesel derivada do óleo de algodão, 16 para soja e 16 para girassol. As amostras do conjunto de teste foram selecionadas utilizando o algoritmo de Kennard-Stone no programa computacional Matlab, para evitar extrapolação do espaço amostral para os espectros do conjunto de teste, sendo composto por $28 \mathrm{me}-$ didas espectrais, em que 6 foram do biodiesel derivado do óleo de algodão, 11 para girassol e 11 para soja. Os modelos foram gerados usando validação cruzada completa, leave-one-out, e o número de PC para os modelos de girassol e algodão foi igual a 3 e soja 4, que foi sugerido pelo próprio software. O número de $\mathrm{PC}$ escolhidos se baseia na curva de variância residual dos modelos, que é a média quadrática dos resíduos de todos os componentes do modelo, sendo que para

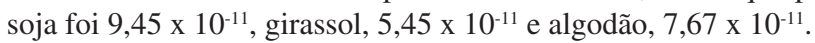

Nos gráficos Si/S0 vs Hi dos modelos SIMCA para as amostras de biodiesel, Figura 4, é possível verificar as distâncias ortogonais das amostras dos conjuntos de teste, indicando os limites para classificação dentro de cada classe, a 95\% de confiança estatística. Os gráficos mostram os dois limites utilizados para classificar uma amostra a um dado modelo, em que Si/S0 é a distância relativa de amostras ao modelo por meio do desvio padrão residual e o leverage (Hi) é a distância da nova amostra ao centro do modelo, que indica o nível de influência da amostra no modelo. Vale ressaltar que a presença de uma amostra de girassol e uma de algodão, distantes de seus grupos e apresentadas na extremidade direita superior dos gráficos, apresentam a variabilidade das amostras nos modelos, não implicando, contudo, em erro de classificação dessas amostras.

Outrossim, a viabilidade em se fazer a classificação das amostras de biodiesel é possível, pois existem diferenças significativas dos ácidos graxos presentes e de suas proporções nos óleos precursores
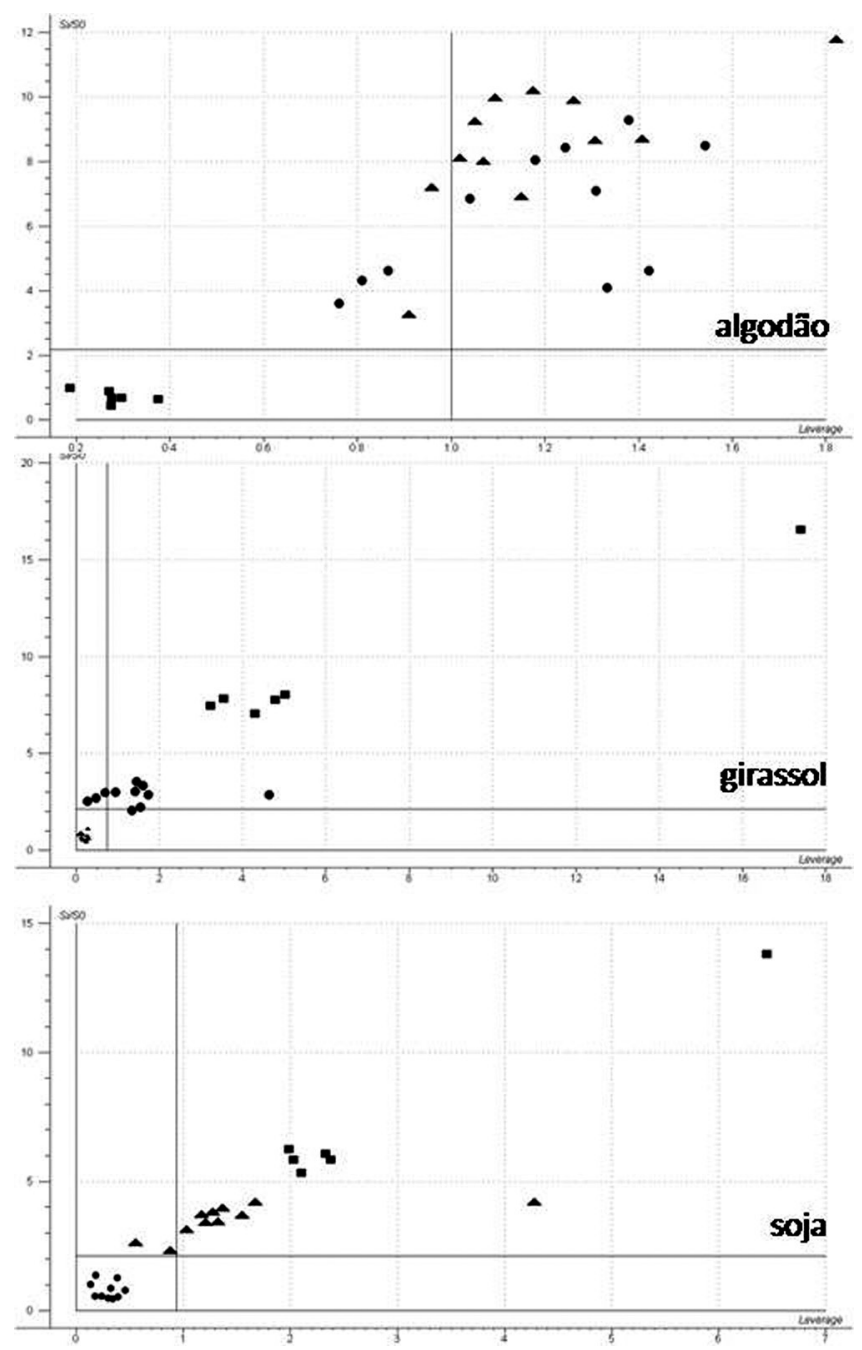

Figura 4. Gráfico de Si/SO vs leverage (Hi) para o grupo de amostras de teste, com base no modelo de algodão ( $\mathbf{\square})$, girassol ( $\mathbf{\Delta}$ ) e soja (-), com $95 \%$ de confiança estatística

e porque as estruturas dos ésteres de ácidos graxos no biodiesel não são distintas dos ácidos graxos dos óleos quando da reação de transesterificação. Da mesma forma, as similaridades existentes entre os biodieseis de soja e girassol e as dissimilaridades entre estes e o biodiesel derivado do óleo de algodão também estão fundamentadas na composição dos ácidos graxos dos seus óleos precursores, corroborando os resultados encontrados neste trabalho. Assim, foi possível, neste trabalho, obter um algoritmo SIMCA para classificação de biodiesel com espectroscopia de absorção molecular na região do visível sem erros de tipo I ou de tipo II.

\section{CONCLUSÕES}

Neste trabalho foram classificadas, sem erros de tipo I e de tipo II, amostras de biodiesel, utilizando técnicas de reconhecimento de padrões e a espectrometria de absorção molecular na região do visível, baseada na diferença de composição e proporções dos ácidos graxos dos óleos precursores, que representam quase $90 \%$ da produção nacional de biodiesel. A possibilidade de implementar esta metodologia na construção de fotômetros dedicados baratos e com componentes de fácil aquisição na classificação de biodiesel para suporte aos órgãos fiscalizadores, às empresas que comercializam e aos consumidores, aponta a relevância deste trabalho. Entretanto, a adição de corantes ou 
de outros aditivos coloridos e a presença de contaminantes nos óleos podem proporcionar erros de classificação, falseando os resultados, que podem ser corrigidos com uma lavagem de biodiesel adequada.

Por fim, a metodologia proposta neste artigo mostrou-se satisfatória para classificação de biodiesel, de forma rápida e não destrutiva, podendo ser empregada no controle de qualidade na forma de análise do tipo screening.

\section{AGRADECIMENTOS}

Ao CNPq pelas bolsas de Iniciação Tecnológica e Industrial e pelo financiamento do projeto $n^{\circ}: 576416 / 2008-8$, ao Dr. E. P. de Medeiros e à Embrapa Algodão pela cessão do espectrômetro.

\section{REFERÊNCIAS}

1. Véras, G.; Gomes, A. A.; Silva, A. C.; Brito, A. L. B.; Almeida, P. B. A.; Medeiros, E .P.; Talanta 2010, 83, 565.

2. Lei $N^{\circ} 11.097,13 / 1 / 2005$ - dispõe sobre a introdução do biodiesel na matriz energética brasileira; altera as Leis n ${ }^{\circ} \mathrm{s} 9.478$, de 6/8/1997, 9.847, de 26/10/1999 e 10.636, de 30/12/2002; e dá outras providências; Diário Oficial da União, 14/1/2005, seção 1, p. 8.
3. Knothe, G.; Gespen, J. V.; Krahl, J.; Manual de biodiesel, Edgard Blücher: São Paulo, 2006.

4. Balabin, R. M.; Safieva, R. Z.; Anal. Chim. Acta 2011, 689, 190.

5. Araújo, M. C. U.; Santos, S. R. B.; Véras, G.; Lima, J. L. F. C.; Lapa, R. A. S.; Quim. Nova 1997, 20, 137.

6. Acrevedo, F. J.; Javier, J.; Maldonado, S.; Domínguez, E.; Narvaez, A.; J. Agric. Food Chem. 2007, 55, 6842.

7. Urbano, M.; Castro, M. D. L.; Pérez, P. M.; García-Olmo, J.; GómezNieto, M. A.; Food Chem. 2006, 97, 166

8. Xing, J,; Ngadi, M,; Gunenc, A,; Prasher, S,; Gariepy, C.; J. Food Eng. 2007, 82, 135.

9. Casale, M.; Armanino, C.; Casolino, C.; Forina, M.; Anal. Chim. Acta 2007, 589, 89.

10. Holler, F. J.; Skoog, D. A.; Crouch, S. R.; Princípios de Análise Instrumental, 6 $6^{\text {a }}$ ed, Bookman: Porto Alegre, 2009.

11. Brereton, R. G.; Chemometrics for pattern recognition, Wiley: Chichester, 2009.

12. Correia, P. R. M.; Ferreira, M. M. C.; Quim. Nova 2007, 30, 481.

13. Lôbo, I. P.; Ferreira, S. L. C.; Cruz, R. S.; Quim. Nova 2009, 32, 1596. 